\section{Computer image analysis of ultrasound biomicroscopy of primate accommodation}

RA Schachar ${ }^{1}$ and F Kamangar ${ }^{2}$
${ }^{1}$ Department of Physics, University of Texas at Arlington, Arlington, TX, USA

${ }^{2}$ Computer Science and Engineering Department, University of Texas at Arlington, Arlington, TX, USA

Correspondence: RA Schachar, PO Box 601149, Dallas, TX 75360, USA Tel: + 12146950080 ; Fax: + 12143685970 . E-mail: ron@

2ras.com

Received: 12 March 2004 Accepted: 12 December 2004;

Published online: 8 April 2005

Dr Schachar has a financial interest in the surgical correction of presbyopia

\begin{abstract}
Purpose To assess and correct images of the eye for movements that can confound the evaluation of the presence, direction, and magnitude of intraocular movement of the crystalline lens equator during centrally induced ciliary muscle contraction (accommodation).

Methods Ultrasound biomicroscopic (UBM) video images of a cynomologus monkey crystalline lens were obtained from an independent source. The images, prior to, during, and following electrical stimulation of the Edinger-Westphal (EW) nucleus were compared for evidence of movement of the crystalline lens equator. Extraocular eye movements were assessed by use of objective computer imaging analysis techniques.

Results Extraocular eye movements were identified and reduced by using objective computer imaging analysis techniques to register and realign the corneal images. Highly significant corrections are required to effect corneal realignment. Analysis of paired and registered images from this data source indicates that any movements of the primate lens equator are not detectable when maximum

accommodation was induced by EW stimulation. Conclusions The displacement of the edge of the primate crystalline lens equator during electrically induced contraction of the ciliary muscle is a small displacement phenomenon, only analysable after confounding extraocular movements are removed from the compared images.

Eye (2006) 20, 226-233. doi:10.1038/sj.eye.6701838; published online 8 April 2005
\end{abstract}

Keywords: primate accommodation; computer image analysis; crystalline lens
Introduction

Glasser and Kaufman ${ }^{1}$ have described large inward movements of the equator of the crystalline lens, associated with electrical stimulation of the Edinger-Westphal (EW) nucleus. They interpreted these movements as evidence in support of the Helmholtz's theory of accommodation. ${ }^{2-4}$

We have been concerned that these large lens displacements might be the result of confounding extraocular movements induced by the periocular muscles during stimulation of the EW nucleus. These ocular movements could mask the assessment and identification of true intraocular movement of the crystalline lens equator induced by contraction of the ciliary muscle. By assessing for the presence of the extraocular movement in images of the cornea, an accurate correction of such extraocular movement can be made. Thereafter, comparison of the realigned images makes an accurate assessment of the actual displacement of the lens equator due to ciliary muscle contraction possible.

We have re-examined the ultrasound biomicroscopic (UBM) video images from their experiments. We used computer image analysis techniques to remove the extraocular movements of the eye relative to the UBM imaging device. This permitted the accurate characterization of any movement of the crystalline lens equator exclusively due to ciliary muscle contraction during EW stimulation.

\section{Methods}

Using a copy of the original UBM video images as provided to us by Glasser and Kaufman, ${ }^{1}$ we analysed the images they obtained of the crystalline lens equator of a cynomolgus 
monkey before, during and after EW stimulation. Kaufman and Glasser adhered to the ARVO standards for the use of animals in ophthalmic and vision research. The cynomolgus monkey studied was 13 years old. The 13-year-old cynomolgus monkey had two recording sessions, separated by at least 25 days. Each recording session involved up to 100 or more stimulus cycles as defined below.

As described by Glasser and Kaufman, ${ }^{1}$ central electrically stimulated accommodation was induced through an electrode that was permanently implanted in the EW nucleus of a totally iridectomized cynomolgus monkey. In order to reduce eye movement, botulinum toxin was injected into the medial and lateral rectus muscles prior to the experimental session. During the session, tension was applied to sutures that were attached to these muscles to attempt to further tamponade the extraocular eye movements.

A stimulus cycle consisted of the application of the electrical stimulus, on phase, to the EW nucleus continuously for 2.2 to $2.5 \mathrm{~s}$, followed by a rest, off phase, for a minimum of $3 \mathrm{~s}$. During each stimulus cycle, the channel marker recorded on the video images indicated $\mathrm{CH} 11$ when the electrical stimulus was in the on phase, and $\mathrm{CH} 00$ when it was in the off phase. In addition, a simultaneous audio signal identified that the electrical stimulus was in the on phase, when the channel marker indicated $\mathrm{CH} 11$.

Ocular movements during each of these stimulus cycles were recorded using a UBM (Zeiss Humphrey Instruments, San Leandro, CA, USA, Model 840), which simultaneously captured the cornea and the equatorial edge of the crystalline lens on videotape at 30 frames/s. The crystalline lens equator and a large portion of the cornea were visible on every image that was employed in our analysis.

The video track provided by Glasser and Kaufman ${ }^{1}$ contained time codes indicating only the hour and minute. The individual frames were not sequentially labelled. We added a continuous time code to the right upper corner of their video track so that each frame was sequentially identifiable.

Software, developed to address the movement artifact associated with positional changes between an imaging device and the object of regard, was employed in our analysis. ${ }^{5}$ The Matlab ${ }^{5}$ program is able to register images, which may have changed in size, shape, or position due to movement artifact during data collection. This software is able to adjust the size and shape of each discrete, video image so that it can be registered to other images with regard to fixed reference points. For our analysis, this permits identification and correction of any changes in the position of the eye induced by extraocular movements. Once the corneal images of any two images are registered, the presence and direction of any change of the intraocular position of the equator of the crystalline lens can be assessed, measured, and recorded.

This study involved registration of images of the same eye that were taken at different times. In this approach, control points on the cornea in the reference image were mapped to the corresponding control points on the cornea in another image. Six corneal control points on each image were selected manually to define the boundaries of the corneal profile. Four points were placed on the endothelial surface and two on the epithelial surface.

An affine transform ${ }^{6,7}$ was used to map these corneal control points of the stimulated image to those of the reference corneal image. Affine transformations are linear and are used to correct for the perspective change that occurs as an imaging device is moved relative to the object. The inverse of the affine transform was employed to identify the pixels in the stimulated image that correspond to those in the reference image. The inverse transform addressed any holes and missing pixels that might appear in the transformation process. The referenced and transformed corneal images are then subtracted from one another. The absolute difference in intensity of the subtracted corneal images is a measure of the processing error associated with the alignment of the corneal images.

In order to align the corneal images, it is necessary to identify corneal registration locations common to both images for the computer comparisons. These boundary points on the corneal images are manually selected. This subjective function was performed in the following manner. The first image of the comparison pair was coloured blue, and the second yellow. The images were then superimposed so that a course alignment was effected. Using four times magnification, two topographically identical loci at the same apparent position on the outer surface and four, on the inner surface, of each of the superimposed corneal images were marked. These points were located at the periphery of the cornea so that the entire cross-sectional area of the corneal image was included between them. In addition, two independent marks were placed at the peripheral margin of the crystalline lens equator in each of the two images. Once defined, no marks were changed at any time during the subsequent analysis. The computer used these selected corneal alignment points to register the two images for every paired comparison.

Initially the software program, prior to any transformation, subtracted the first image from the second image. The light intensity of each pixel of every image from each comparison was normalized so that the maximum light intensity of a pixel was equal to 1 . The computer calculated the root mean square of the image intensity of the corneal pixels following an absolute 
subtraction of the first and second images. Then, after a positional and spatial transformation of the second image of the pair, the images were superimposed and subtracted.

The final computational step was to measure and record the distance between the two independent marks at the peripheral margin of the crystalline lens equator from the two transformed images. The mean difference in the $x, y$ coordinates of the edge of the lens equator position and its SD were determined for all aligned image pairs in each subgroup. This was the measure of movement of the crystalline lens equator following EW stimulated accommodation.

\section{Controls}

We analysed the ability of the software to realign the corneas from identical images pairs, after one image had been randomly rotated and or linearly displaced with regard to the other. We selected one frame from the off phase and duplicated it. We randomly rotated one image, from $-3.0^{\circ}$ to $+3.0^{\circ}$ in $0.5^{\circ}$ steps, and or displaced it, from -2 pixels to +2 pixels in 1 pixel steps relative to the second identical image. A total of 65 images were duplicated, rotated, and/or displaced, and then compared. This test was repeated on four different randomly selected off phase images. A total of 260 comparisons of identical image pairs underwent random amounts of rotation and/or translation. We thereby obtained a measure of the combined error associated with the process of marking, registering, aligning, and subtracting the transformed corneal images.

\section{Analysis of the paired Same Phase Images, either only 'off phase' or only 'on phase'}

We compared the 5th, 15th and 25th off phase images from each stimulus cycle prior to the electrical stimulation to statistically determine the alignment reproducibility of the same cycle off phase images both before and after Matlab transformation. It was assumed that any movement between these images was due to movement of the eye relative to the UBM. Similarly, the 5 th, $15^{\text {th }}$, and 25th on phase images after the electrical stimulus was on for one full second were compared with the assumption that the ciliary muscle was in the maximally stimulated state during this time interval. The selection of these time points was based upon the time at which the minimum and maximum refractive errors occurred, as reported by Glasser and Kaufman, ${ }^{1}$ (see Figure 5a of their paper).

\section{Analysis of the paired off and on Phase images}

We employed these same stimulus cycles as those used by Glasser and Kaufman ${ }^{1}$ in their published images. Data came from the 1-min interval of their videotape: 12:44:00 to $12: 44: 54$ (h:min:sec) on the 7 July 1997 session. Examination of their published images, which are reproduced herein, reveals that they compared images in the same phase. (Note that both channel markers in Figure 1(a) and (b) of their paper are $\mathrm{CH} 11$, indicating that both of these images were taken from the same on phase. Similarly, note that both channel markers in Figure $1(\mathrm{~d})$ and (e) of their paper are $\mathrm{CH} 00$, indicating that both of these images where taken from the same off phase) (Figure 1).

We re-evaluated their published images Figures 1d and e, (Figure 1). We were unable to re-evaluate their published images Figures $1 \mathrm{a}$ and $\mathrm{b}$ because they were over exposed and incorporated the conjunctiva, which precluded registration using the sclera and the cornealscleral junction.

Using the sequential frame codes, we were able to reanalyse all five stimulus cycles that occurred during the 1-min time interval that they reported in their paper. It was not possible to distinguish which of the five stimulus cycles in that minute they used due to the absence of sequential codes on their video. Comparisons were made between each of the three images in the off phase with each of the three in the on phase for each stimulus cycle, making for a total of nine comparisons per stimulus cycle. A total of 45 comparisons were made (nine comparisons per stimulus cycle, for five stimulus cycles).

In order to maximize the data analysis, we also analysed five consecutive stimulus cycles from the second session of the same 13-year-old monkey from Glasser and Kaufman's videotape from the 1-min interval: 11:59:00 to 12:00:00 on the 11 June 1997 session. During the off phase of each cycle, the 5th, 15th, and 25th images occurring in the 1-s interval just prior to EW electrical stimulation were identified. During the on phase, the 5th, 15th, and 25th images occurring in the onesecond interval after the onset of stimulation, were identified. As noted previously, these time intervals corresponded to the minimum and maximum refractive change following the EW electrical stimulation. ${ }^{1}$

Comparisons were made between each of the three images in the off phase with each of the three in the on phase for each stimulus cycle, making for a total of nine comparisons per stimulus cycle. A total of 45

comparisons were made (nine comparisons per stimulus cycle, for five stimulus cycles).

Typical off phase, $\mathrm{CH} 00$, and on phase images, $\mathrm{CH} 11$, and the superposition of the two images that were used to make the comparison are shown in Figure 2a-c, respectively (Note the $\mathrm{CH} 00$ in (a) and $\mathrm{CH} 11$ in (b), indicating that (a) was in the off phase, and that (b) the second image, was in the on phase. The greater than $1.5 \mathrm{~s}$ difference in the time codes between (a) and (b), shown 

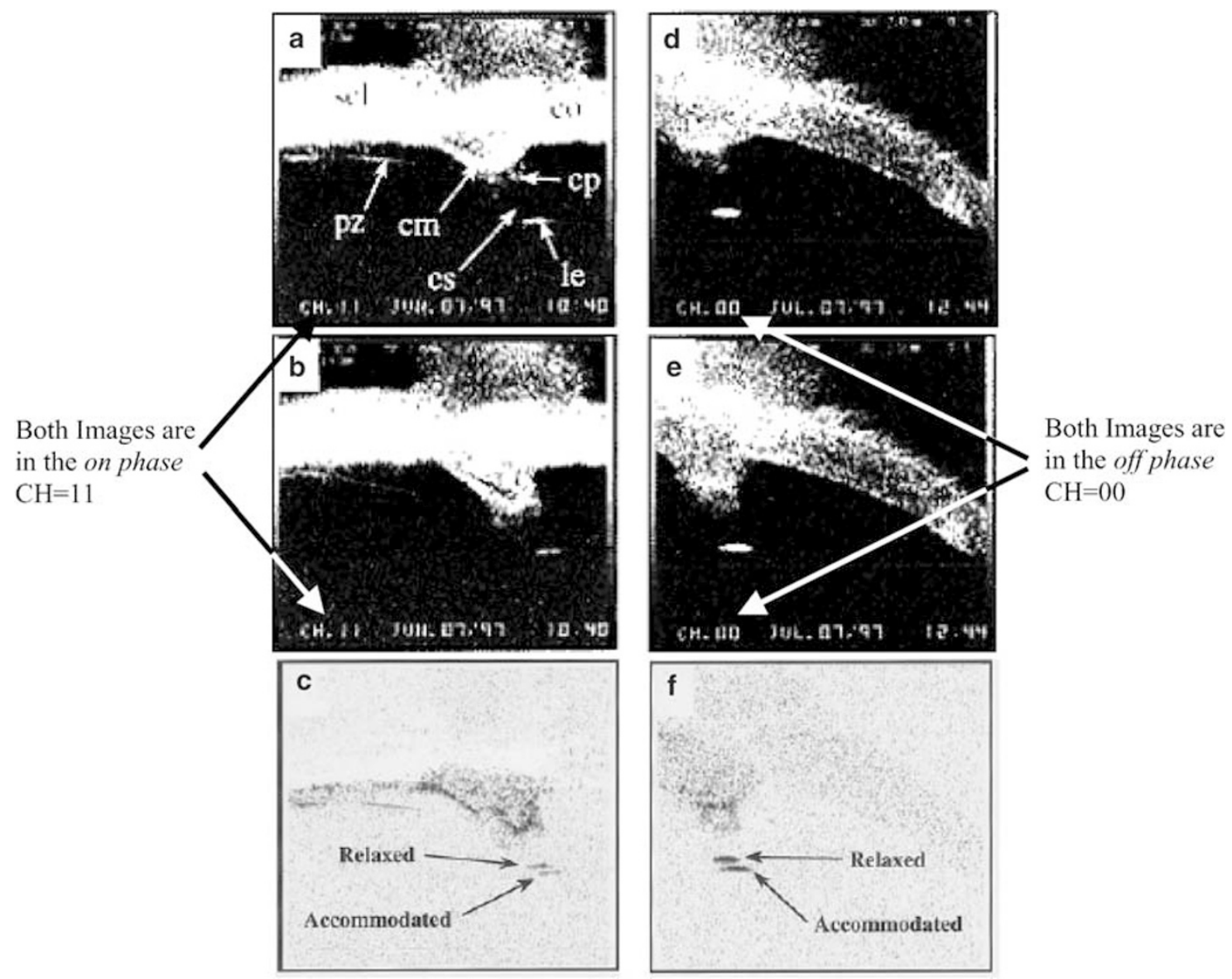

Figure 1 Glasser and Kaufman's Figures 1a-e, ${ }^{1}$ reprinted with permission from the American Academy of Ophthalmology, include the following legend:

"Ultrasound biomicroscopic images of the temporal ciliary region of an iridectomized $\sim 13$-year-old cynomolgus monkey eye on two separate occasions. Accommodation was induced by electrical stimulation of the midbrain. $\mathrm{Scl}=\mathrm{sclera}$; $\mathrm{CO}=\mathrm{cornea}$; $\mathrm{PZ}=$ posterior zonule; $\mathrm{CM}=$ ciliary musde; $\mathrm{CS}=$ circumlental space; $\mathrm{LE}=$ lens equator; $\mathrm{CP}=$ ciliary processes. The equatorial edge of the lens, visible as a short horizontal line, reflects ultrasound back to the transducer, but the anterior zonular fibers and the anterior and posterior lens surfaces do not and thus are not visible. (a) the temporal sclera and underlying ciliary muscle are seen in the unaccommodated eye. The posterior zonular fibres extend between the inner apex and posterior attachment of the ciliary muscle. (b) In the accommodated state, the inner apex of the ciliary muscle moves forward and toward the axis of the eye. The posterior zonular fibres are stretched, and the equatorial edge of the lens moves away from the sclera and toward the anterior-posterior axis. (c) The subtracted image pairs show a virtual absence of eye movement. Dark areas show movement of structures between images; white areas are stable. The only movement visible is of the ciliary muscle and lens equator. The lens equator has moved $\sim 100 \mu \mathrm{m}$ away from the sclera. (d) unaccommodated, (e) accommodated, and (f) subtracted image pairs from the temporal side of the same animal on another occasion."

Note that in contrast to Glasser and Kaufman's legend the compared images are in the same phase.

in the right upper part of the image, verifies that the central electrical stimulus had been continuously applied for a minimum of $1.0 \mathrm{~s}$. Thus, (b) was obtained following maximal stimulation).

\section{Results}

Transformation of intentionally displaced identical images (identical controls) improves corneal registration. The mean induced displacement was 0.051 normalized intensity units before transformation and 0.028 normalized intensity units after, a difference of 0.023 normalized intensity units, $P<0.0001$, Table 1 . Bland Altman plots of these paired control images visually demonstrate the reduction in variability of the lens equatorial position following transformation of each of the paired images, $P<0.0001$ (Figure 3a). Bland Altman plots of identical paired images, one of which was rotated and/or displaced, demonstrate that the computer image transformation reduced the range of positional variability of the lens equator by 2.5 times (Figure 3a). The paired off phase image (off phase controls) were 
a

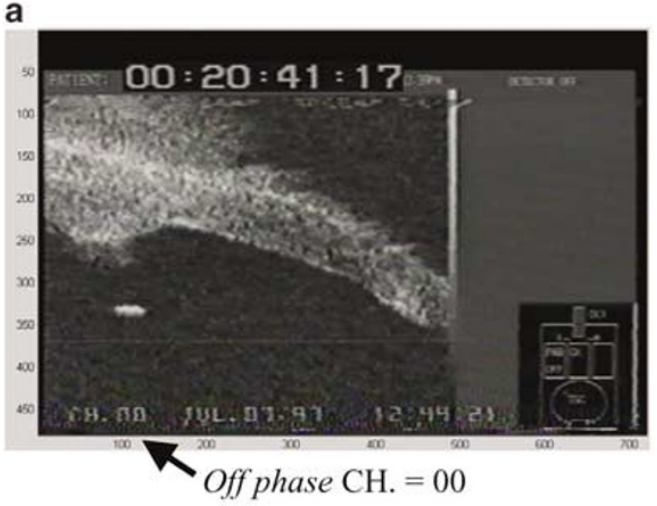

b

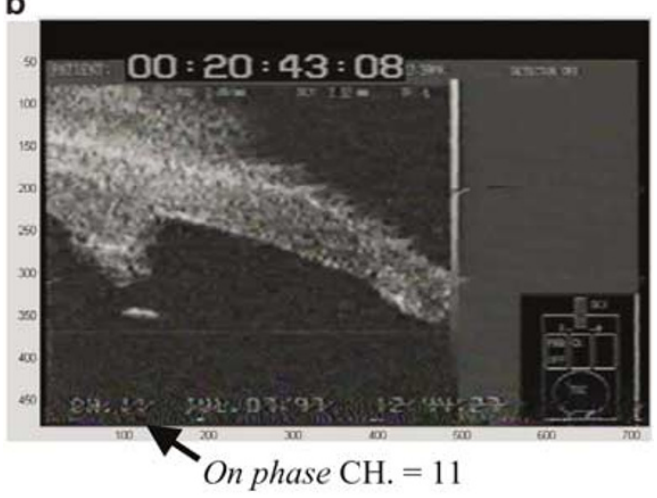

C

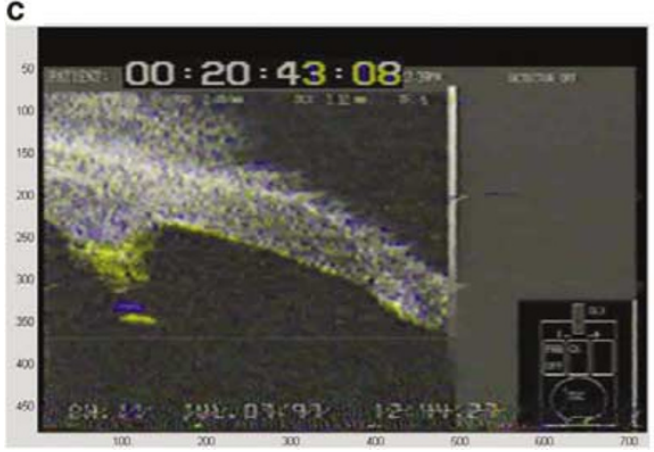

Figure 2 UBM images of the cornea and crystalline lens equator of a 13-year-old cynomolgus monkey in the (a) relaxed state, $0.17 \mathrm{~s}$ before initiation of the EW electrical stimulation; $(\mathrm{b})$ stimulated state, $1.5 \mathrm{~s}$ after initiation of the EW electrical stimulation; (c) superposition of (a) (blue) and (b) (yellow). relatively stable, as indicated by the vertical lens equatorial location remaining within \pm 2.8 pixels following transformation (Figure $3 b$ ). However, the paired on phase images (on phase controls) reveal substantial eye movement, reflected by a larger SD, which is reduced following transformation. (Figure 3c). Note the mean change in the lens equator position of nearly 2 pixel units from the same on phase images before transformation. This artifactious movement is completely removed by the transformation, as the mean vertical lens equatorial location becomes zero (Figure $3 \mathrm{c}$ ).

Computer subtraction of the off and on phase images from the stimulus cycle of Glasser and Kaufman's session 1 demonstrates the lack of registration of the corneal images from the off and on phases (Figure 4).

There was obvious extraocular eye movement relative to the UBM between these two images. Glasser and Kaufman ${ }^{1}$ did not compare these two images, but selected images from the same off phase for comparison in their paper (Figure 1).

Computer transformation significantly improved corneal registration of the images from both session 1 and 2, $P<0.0001$ (Table 2, Figure 5). The analysis for a shift in the equatorial position with EW stimulation after image pair transformation indicates that only a minimal vertical movement of 0.6 pixels toward the sclera occurred, $P=0.04$ (Table 3). There was no statistically demonstrable movement in the horizontal direction, $P=0.23$.

\section{Discussion}

This paper has provided a methodology for assessing and correcting for confounding eye movements associated with serial image capture and stimulation of the EW nucleus. This is an essential study control required before it is possible to assess any lenticular movement due to ciliary muscle stimulation. Glasser and Kaufman ${ }^{1}$ attempted to reduce the eye movement by using traction sutures, and injecting botulinum toxin into the medial and lateral rectus muscles. However, this procedure left residual movement demonstrated by examination of the video images. Purkinje image I of

Table 1 Comparison of alignment of displaced identical corneal images before and after computer image transformation

\begin{tabular}{|c|c|c|c|c|c|}
\hline \multicolumn{6}{|c|}{$\begin{array}{l}\text { Identical controls } \\
\text { Mean corneal alignment differences (in normalized intensity units) }\end{array}$} \\
\hline Procedure & $\begin{array}{l}\text { Number of } \\
\text { compared } \\
\text { Image pairs }\end{array}$ & $\begin{array}{l}\text { Original } \\
\text { minus } \\
\text { displaced }\end{array}$ & $\begin{array}{c}\text { Original } \\
\text { minus } \\
\text { transformed displaced }\end{array}$ & $\begin{array}{c}\text { Difference (original-displaced) } \\
\text { minus } \\
\text { (original-transformed displaced) }\end{array}$ & $\begin{array}{l}\text { Probability } \\
\text { (two-tailed } \\
\text { " } \mathrm{t} \text { "-test) }\end{array}$ \\
\hline $\begin{array}{l}\text { Rotated and/ } \\
\text { or translated }\end{array}$ & 260 & $0.051 \pm 0.019$ & $0.028 \pm 0.023$ & $0.023 \pm 0.021$ & $<0.0001$ \\
\hline
\end{tabular}



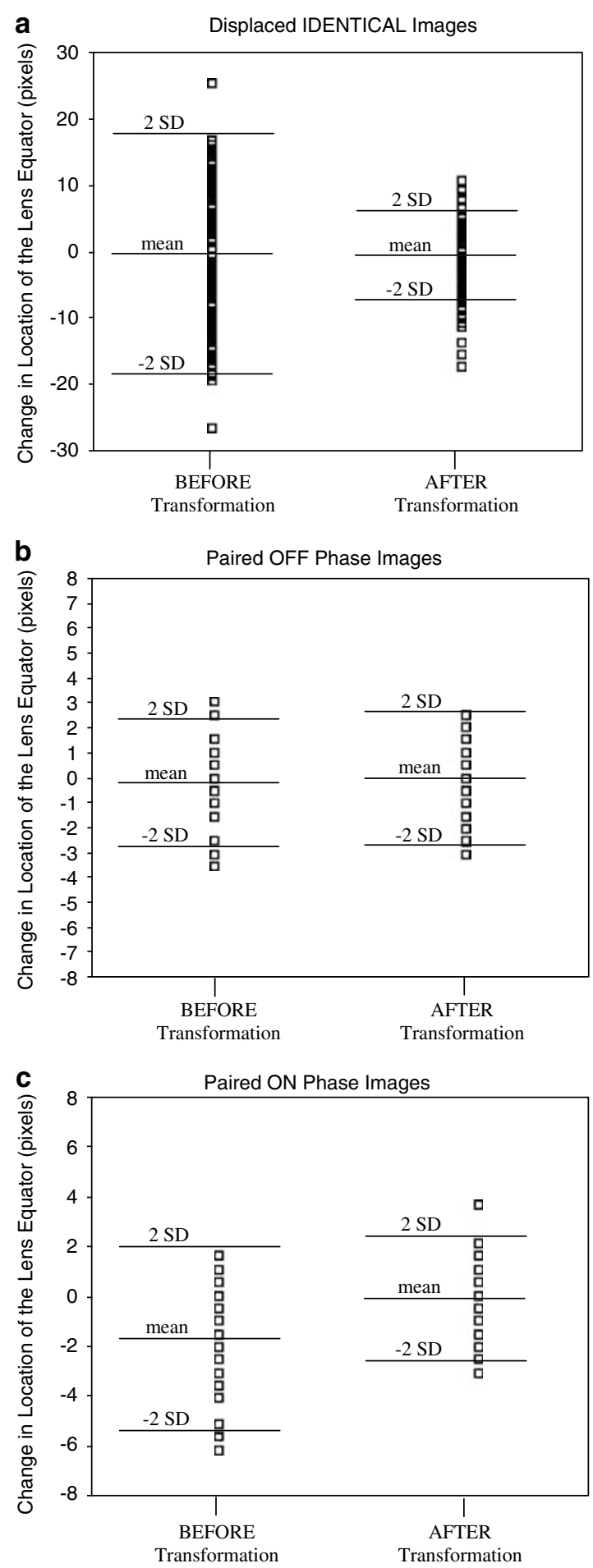

Figure 3 Bland Altman plotting of the change in location of the lens equator before and after computer transformation: (a) displaced identical images; (b) paired off phase images; (c) paired on phase images (plus values indicate that the location of the lens equator moved vertically toward the sclera).

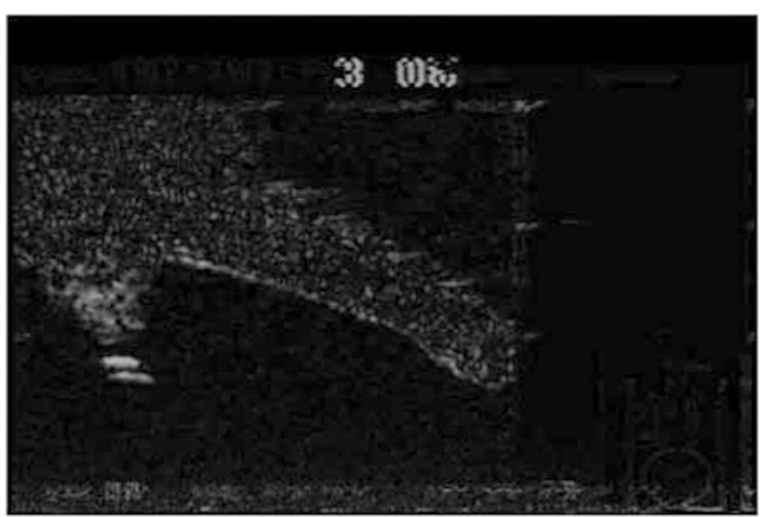

BEFORE TRANSFOMATION

Figure 4 Shift in position of Lens Equator by subtraction of the relaxed image of Figure $2 \mathrm{a}$ minus the stimulated image of Figure $2 \mathrm{~b}$ without any transformation. Owing to the image subtraction the labels are not readable.

their monkey eyes revealed vertical and torsional movements during EW stimulated accommodation, probably due to unrestricted contraction of the oblique extraocular muscles. The UBM has a resolution of $12 \mu \mathrm{m}^{8}$ so that small movements of the eye between images relative to the position of the UBM can be recorded, but may be misinterpreted. It is clear that without proper ocular alignment controls and corrections for extraocular eye movements, assessment of these images for lenticular movement is hopelessly confounded.

Total control of eye movement relative to the imaging device, in vivo, requires an accurate and precise $x, y, z$ tracker. In the absence of such a tracker, we used computer image analysis to correct for movement of the eye relative to the imaging device. Similar types of postprocessing computer image analysis have been used successfully to correct for motion artifact that occurs during magnetic resonance imaging (MRI)., ${ }^{9,10}$

This method depends upon using the cornea as a fixed reference from which to register and transform images before making measurements of the relative position of the lens equator during accommodation. There have been reports of corneal topographic changes during accommodation. ${ }^{11,12}$ Measurement of the accommodative amplitude, while optically removing the cornea as a refractive surface, has demonstrated that the cornea does not contribute to the amplitude of accommodation in humans. ${ }^{13,14}$ Measurement of the position of the cornea with either $\mathrm{UBM}^{8}$ or partial coherent interferometry ${ }^{15}$ (PCI) also has demonstrated that the cornea does not change shape or position during accommodation. When correction is made for the convergent eye movements that occur with accommodation, a clinically insignificant change of 0.16 diopters and a $2 \mu \mathrm{m}$ change in height was noted. ${ }^{16}$ Furthermore, if correction is made for the excyclotorsional eye movement that occurs during 
Table 2 Comparison of corneal image alignment before and after computer image transformation during EW stimulation

\begin{tabular}{|c|c|c|c|c|c|}
\hline \multicolumn{6}{|c|}{$\begin{array}{l}\text { EW stimulated } \\
\text { Mean corneal alignment differences (in normalized intensity units) }\end{array}$} \\
\hline $\begin{array}{l}\text { EW } \\
\text { stimulation }\end{array}$ & $\begin{array}{l}\text { Number of compared } \\
\text { image pairs }\end{array}$ & Off-on & Off-transformed on & $\begin{array}{l}\text { Difference [off-on] minus } \\
\text { [off-transformed on] }\end{array}$ & $\begin{array}{l}\text { Probability (two- } \\
\text { tailed " } \mathrm{t} \text { " test) }\end{array}$ \\
\hline Session 1 & 45 & $0.086 \pm 0.005$ & $0.078 \pm 0.002$ & $0.008 \pm 0.004$ & $<0.0001$ \\
\hline Session 2 & 45 & $0.113 \pm 0.008$ & $0.0108 \pm 0.005$ & $0.005 \pm 0.004$ & $<0.0001$ \\
\hline
\end{tabular}

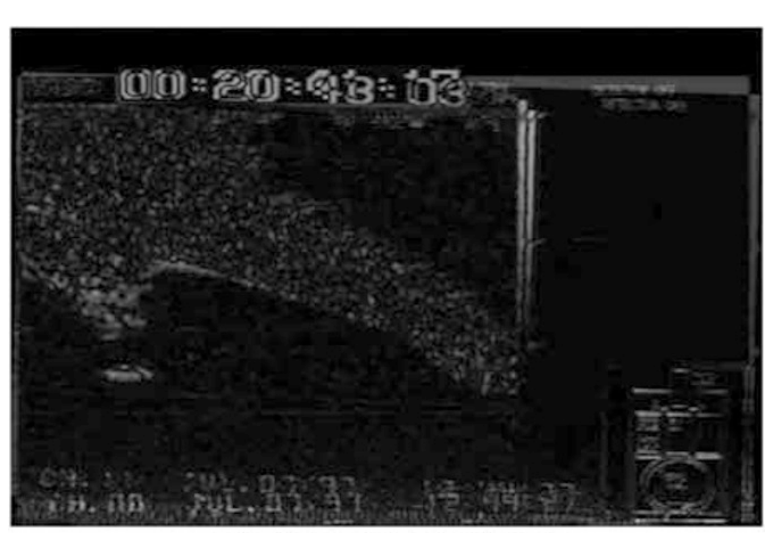

AFTER TRANSFORMATION

Figure 5 Shift in position of Lens Equator by subtraction of the relaxed image of Figure $2 \mathrm{a}$ minus the stimulated image of Figure $2 b$ with image transformation. Owing to the image subtraction the labels are not readable.

accommodation, no topographical changes are found. ${ }^{17}$ Therefore, the human cornea is stable during accommodation. The primate cornea, which is anatomically similar, is believed to also be stable during accommodation and was used exclusively as the fixed reference for the alignment of all UBM images. Neither the ciliary body nor the zonules were used in any way as positional references for this analysis.

Since the UBM images are two-dimensional, the computer image analysis only corrects for movements in the $x-y$ plane. Movements in the $z$ direction may be associated with additional measurement errors that cannot be corrected by the present analysis. However, if spurious eye movements in the $z$-direction were large, they would produce two-dimensional configurational changes in the cornea, which might be detectable. None were noted.

Bland Altman analysis of paired control images, be they identical, off phase, or on phase pairs, demonstrates the reduction in variability of the mean change of position of the lens equator after transformation, when compared to the same-paired images before transformation (Figure 3). There is a marked improvement in the localization of the lens equator in the paired on phase controls after computer image transformation, indicated by a mean change from -2 to zero pixels. The off phase controls are much more stable, with little change in the position of the equator following transformation.

Analysis of the paired off and on phase images demonstrated that with approximately 15 diopters of lens accommodation, the lens equator moved less than the resolution of this measurement system (Figure 5). We were unable to quantitate the number of microns / diopter that the crystalline lens equator moved because the displacement was below or at the threshold of the measurement technique. However, UBM imaging of the changes in the position of the crystalline lens equator of cynomolgus monkeys following maximum pharmacologically induced accommodation of 30 diopters, demonstrated a $2 \mu \mathrm{m}$ /diopter movement. ${ }^{18}$ This is consistent with our present analysis. It is clear that

Table 3 Comparison of equatorial lens position before and after computer image transformation during EW stimulation

\begin{tabular}{|c|c|c|c|c|c|c|c|c|c|}
\hline \multirow{3}{*}{$\begin{array}{c}E W \\
\text { stimulation }\end{array}$} & \multicolumn{7}{|c|}{$\begin{array}{c}E W \text { stimulated } \\
\text { Mean differences in the location of the lens equator (pixels) }\end{array}$} & & \\
\hline & \multirow[t]{2}{*}{$\begin{array}{c}\text { Number of } \\
\text { compared } \\
\text { images pairs }\end{array}$} & \multicolumn{2}{|c|}{ Off-on } & \multicolumn{2}{|c|}{ Off-transformed on } & \multicolumn{2}{|c|}{$\begin{array}{l}\text { Probability [off-on] minus } \\
\text { [off-transformed on] } \\
\text { (two-tailed " } \mathrm{t} \text { " test) }\end{array}$} & \multicolumn{2}{|c|}{$\begin{array}{c}\text { Probability } \\
\text { [off-transformed on] } \\
\text { (two-tailed " } \mathrm{t} \text { " test) }\end{array}$} \\
\hline & & Vertical & Horizontal & Vertical & Horizontal & Vertical & Horizontal & Vertical & Horizontal \\
\hline Sessions $1 \& 2$ & 90 & $-16.1 \pm 3.0$ & $-11.9 \pm 2.7$ & $0.6 \pm 2.8$ & $-0.6 \pm 5.0$ & $<0.0001$ & $<0.001$ & 0.04 & 0.29 \\
\hline
\end{tabular}

Plus vertical values indicate the lens equator moved vertically towards the sclera, and negative horizontal values indicate that the lens equator moved horizontally towards the cornea. 
movement of the crystalline lens equator during accommodation is in this small displacement range.

Mathematical analysis has demonstrated that accommodation must be a small displacement phenomenon in order to occur within the physiological force range of the ciliary muscle. ${ }^{19-23}$ These same models also demonstrated that as zonular traction is applied to the unaccommodated lens, the central surface steepens, and the central thickness and optical power increase. Consistent with the present study and these mathematical predictions, when zonular tension is applied to the unaccommodated lens, either in vitro ${ }^{24-27}$ or in $v i v o^{8,18}$ to animal or human lenses, the central anterior surface steepens.

In view of the small displacement of the lens equator, any eye movement relative to the imaging device that is large will confound the true direction and positional change of the equator of the crystalline lens and can be misinterpreted as due to accommodation. This is an error that has repeatedly occurred in the evaluation of accommodation. ${ }^{19}$

Future accommodation experiments must take into consideration potential eye movements relative to the imaging device. The use of computer image analysis techniques to compensate for movement of the eye relative to the UBM allows for the identification of extraocular induced movement artifact and its correction. Future studies will require higher resolution and should incorporate proper controls for extraocular movement, including fixed reference points from which to make small accurate precise measurements, three-dimensional eye tracking, image triangulation, high-resolution instrumentation that has been extensively validated, and exact image registration.

\section{Acknowledgements}

We wish to thank Adrian Glasser, PhD and Paul L Kaufman, MD for supplying the videotapes for analysis.

\section{References}

1 Glasser A, Kaufman PL. The mechanism of accommodation in primates. Ophthalmology 1999; 106: 863-872. Medline.

2 von Helmholtz H. Ueber die akkommodation des auges. Arch Ophthalmol 1855; 1: 1-74.

3 Levy NS. The mechanism of accommodation in primates [letter]. Ophthalmology 2000; 107: 625. Medline.

4 Glasser A, Kaufman PL. The mechanism of accommodation in primates [author reply]. Ophthalmology 2000; 107: 625-626. Medline.

5 MatLab 6 Version 1. The MathWorks, Inc.: Natick, MA, 2002.
6 Fitzpatrick JM, West JB, Maurer CR. Predicting error in rigid-body point-based registration. IEEE Trans Med Imag 1998; 17: 694-702. Medline.

7 Maintz J, Viergever MA. A survey of medical image registration. Med Image Anal. 1998; 2: 1-36. Medline.

8 Schachar RA, Tello C, Cudmore DP, Liebmann JM, Black TD, Ritch R. In vivo increase of the human lens equatorial diameter during accommodation. Am J Physiol. 1996; 271(3 Part 2): R670-R676. Medline.

9 Hajnal JV, Saeed N, Soar EJ, Oatridge A, Young IR, Bydder GM. A registration and interpolation procedure for subvoxel matching of serially acquired MR images. J Comput Assist Tomogr 1995; 19: 289-296. Medline.

10 Maas L, Frederick B, Renshaw P. Decoupled automated rotational and translational registration for functional MRI time series data: the DART registration algorithm. Magn Reson Med 1997; 37: 131-139. Medline.

11 Löpping H, Weale RA. Changes in corneal curvature following ocular convergence. Br J Physiol Opt 1959; 5: 207-215.

12 Yasuda A, Yamaguchi T, Ohkoshi K. Changes in corneal curvature in accommodation. J Cataract Refract Surg 2003; 29: 297-301. Medline.

13 Young T. On the mechanism of the eye. Philos Trans Roy Soc (London) 1801; 92: 23-88.

14 Westheimer $\mathrm{G}$. The visual world of the contact lens wearer. J Amer Optom Ass 1962; 34: 135-138.

15 Drexler W, Baumgartner A, Findl O, Hitzenberger CK, Fercher AF. Biometric investigation of changes in the anterior eye segment during accommodation. Vis Res 1997; 37: 2789-2800. Medline.

16 He JC, Gwiazda J, Thorn F, Held R, Huang W. Change in corneal shape and corneal wave-front aberration with accommodation. J Vis 2003; 7: 456-463. Medline.

17 Buehren T, Collins MJ, Loughridge J, Carney LG, Iskander DR. Corneal topography and accommodation. Cornea 2003; 22: 311-316. Medline.

18 Schachar RA, Black TD, Kash RL, Cudmore DP, Schanzlin DJ. The mechanism of accommodation and presbyopia in the primate. Ann Ophthalmol 1995; 27: 58-67. Paper.

19 Schachar RA, Bax AJ. Mechanism of human accommodation as analyzed by non-linear finite element analysis. Comp Ther 2001; 33: 122-132. Medline.

20 Burd HJ, Judge SJ, Cross JA. Numerical modeling of the accommodating lens. Vis Res 2002; 42: 2235-2251. Medline.

21 Chein $\mathrm{CH}$, Huang T, Schachar RA. A model for crystalline lens accommodation. Compr Ther 2003; 29: 167-175. Medline.

22 Shung WV. An analysis of a crystalline lens subjected to equatorial periodic pulls. PhD. thesis University of Texas at Arlington: Arlington, TX, 2002.

23 Chein $\mathrm{CH}$, Huang T, Schachar RA. Analysis of human crystalline lens accommodation. J Biomech, (in press).

24 Stadfeldt, AE. Die veränderung der lines bei traction der zonula. Klinische Monatsblatten für Augenheilkunde 1896; 34: 429-431.

25 Pierscionek B. In vitro alteration of human lens curvatures by radial stretching. Exp Eye Res 1993; 57: 629-635. Medline.

26 Pierscionek B. Personal communication. 2004.

27 Schachar RA. Qualitative effect of zonular tension on freshly extracted intact human crystalline lenses: implications for the mechanism of accommodation. Invest Ophthalmol Vis Sci 2004; 45: 2691-2695. Medline. 